\title{
Frequent mutation of histone-modifying genes in non-Hodgkin lymphoma
}

Ryan D. Morin ${ }^{1 *}$, Maria Mendez-Lago ${ }^{1 *}$, Andrew J. Mungall ${ }^{1}$, Rodrigo Goya ${ }^{1}$, Karen L. Mungall $^{1}$, Richard D. Corbett ${ }^{1}$, Nathalie A. Johnson ${ }^{2}$, Tesa M. Severson ${ }^{1}$, Readman Chiu ${ }^{1}$, Matthew Field ${ }^{1}$, Shaun Jackman ${ }^{1}$, Martin Krzywinski ${ }^{1}$, David W. Scott ${ }^{2}$, Diane L. Trinh ${ }^{1}$, Jessica Tamura-Wells ${ }^{1}$, Sa Li $^{1}$, Marlo R. Firme ${ }^{1}$, Sanja Rogic ${ }^{2}$, Malachi Griffith ${ }^{1}$, Susanna Chan ${ }^{1}$,

Oleksandr Yakovenko ${ }^{1}$, Irmtraud M. Meyer ${ }^{3}$, Eric Y. Zhao ${ }^{1}$, Duane Smailus ${ }^{1}$, Michelle Moksa ${ }^{1}$, Suganthi Chittaranjan ${ }^{1}$, Lisa Rimsza ${ }^{4}$, Angela Brooks-Wilson ${ }^{1,5}$, John J. Spinelli ${ }^{6,7}$, Susana Ben-Neriah ${ }^{2}$, Barbara Meissner ${ }^{2}$, Bruce Woolcock ${ }^{2}$, Merrill Boyle $^{2}$, Helen McDonald ${ }^{1}$, Angela Tam ${ }^{1}$, Yongjun Zhao ${ }^{1}$, Allen Delaney ${ }^{1}$, Thomas Zeng ${ }^{1}$, Kane Tse ${ }^{1}$, Yaron Butterfield ${ }^{1}$, Inanç Birol ${ }^{1}$, Rob Holt ${ }^{1}$, Jacqueline Schein ${ }^{1}$, Douglas E. Horsman ${ }^{2}$, Richard Moore ${ }^{1}$, Steven J. M. Jones ${ }^{1}$, Joseph M. Connors ${ }^{2}$, Martin Hirst ${ }^{1}$, Randy D. Gascoyne ${ }^{2,8}$ \& Marco A. Marra ${ }^{1,9}$

Follicular lymphoma (FL) and diffuse large B-cell lymphoma (DLBCL) are the two most common non-Hodgkin lymphomas (NHLs). Here we sequenced tumour and matched normal DNA from 13 DLBCL cases and one FL case to identify genes with mutations in B-cell NHL. We analysed RNA-seq data from these and another 113 NHLs to identify genes with candidate mutations, and then re-sequenced tumour and matched normal DNA from these cases to confirm 109 genes with multiple somatic mutations. Genes with roles in histone modification were frequent targets of somatic mutation. For example, $32 \%$ of DLBCL and $89 \%$ of FL cases had somatic mutations in MLL2, which encodes a histone methyltransferase, and $11.4 \%$ and $13.4 \%$ of DLBCL and FL cases, respectively, had mutations in MEF2B, a calcium-regulated gene that cooperates with CREBBP and EP300 in acetylating histones. Our analysis suggests a previously unappreciated disruption of chromatin biology in lymphomagenesis.

Non-Hodgkin lymphomas (NHLs) are cancers of B, T or natural killer lymphocytes. The two most common types of NHL, follicular lymphoma (FL) and diffuse large B-cell lymphoma (DLBCL), together comprise $60 \%$ of new B-cell NHL diagnoses each year in North America ${ }^{1}$. FL is an indolent and typically incurable disease characterized by clinical and genetic heterogeneity. DLBCL is aggressive and likewise heterogeneous, comprising at least two distinct subtypes that respond differently to standard treatments. Both FL and the germinal centre B-cell (GCB) cell of origin (COO) subtype of DLBCL derive from germinal centre $B$ cells, whereas the activated $B$-cell $(\mathrm{ABC})$ variety, which has a more aggressive clinical course, is thought to originate from $B$ cells that have exited, or are poised to exit, the germinal centre ${ }^{2}$. Current knowledge of the specific genetic events leading to DLBCL and FL is limited to the presence of a few recurrent genetic abnormalities ${ }^{2}$. For example, $85-90 \%$ of FL and $30-40 \%$ of GCB DLBCL cases $^{3,4}$ harbour $\mathrm{t}(14 ; 18)(\mathrm{q} 32 ; \mathrm{q} 21)$, which results in deregulated expression of the BCL2 oncoprotein. Other genetic abnormalities unique to GCB DLBCL include amplification of the $c$-REL gene and of the miR-17-92 microRNA cluster ${ }^{5}$. In contrast to GCB cases, $24 \%$ of ABC DLBCLs harbour structural alterations or inactivating mutations affecting $P R D M 1$, which is involved in differentiation of GCB cells into antibody-secreting plasma cells ${ }^{6}$. ABCspecific mutations also affect genes regulating NF- $\kappa B$ signalling ${ }^{7,8,9}$, with TNFAIP3 (also known as A20) and MYD88 (ref. 10) the most abundantly mutated in $24 \%$ and $39 \%$ of cases, respectively. To enhance our understanding of the genetic architecture of B-cell NHL, we undertook a study to (1) identify somatic mutations and
(2) determine the prevalence, expression and focal recurrence of mutations in FL and DLBCL. Using strategies and techniques applied to cancer genome and transcriptome characterization by ourselves and others ${ }^{11,12,13}$, we sequenced tumour DNA and/or RNA from 117 tumour samples and 10 cell lines (Supplementary Tables 1 and 2) and identified 651 genes (Supplementary Figure 1) with evidence of somatic mutation in B-cell NHL. After validation, we showed that 109 genes were somatically mutated in two or more NHL cases. We further characterized the frequency and nature of mutations within $M L L 2$ and $M E F 2 B$, which were among the most frequently mutated genes with no previously known role in lymphoma.

\section{Identification of recurrently mutated genes}

We sequenced the genomes or exomes of 14 NHL cases, all with matched constitutional DNA sequenced to comparable depths (Supplementary Tables 1 and 2). After screening for single nucleotide variants followed by subtraction of known polymorphisms and visual inspection of the sequence read alignments, we identified 717 nonsynonymous variants (coding single nucleotide variants; cSNVs) affecting 651 genes (Supplementary Figure 1 and Supplementary Methods). We identified between 20 and 135 cSNVs in each of these genomes. Only 25 of the 651 genes with cSNVs were represented in the cancer gene census (December 2010 release) ${ }^{14}$.

We performed RNA sequencing (RNA-seq) on these 14 NHL cases and an expanded set of 113 samples comprising 83 DLBCL, 12 FL and 8 B-cell NHL cases with other histologies and 10 DLBCL-derived cell lines (Supplementary Table 2). We analysed these data to identify

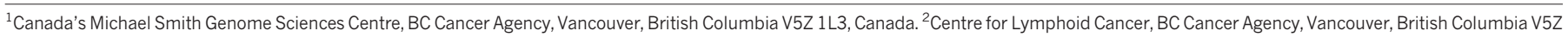

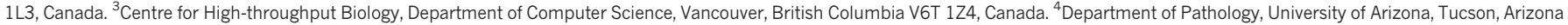

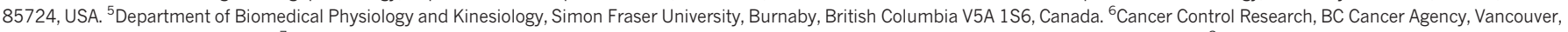

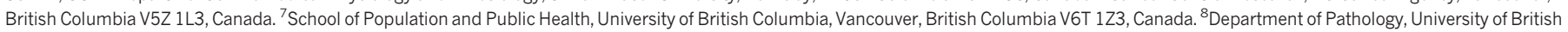
Columbia, Vancouver, British Columbia V6T 2B5, Canada. ${ }^{9}$ Department of Medical Genetics, University of British Columbia, Vancouver, British Columbia V6H 3N1, Canada.

*These authors contributed equally to this work. 
novel fusion transcripts (Supplementary Table 3) and cSNVs (Fig. 1). We identified 240 genes with at least one cSNV in a genome/exome or an RNA-seq 'mutation hot spot' (see later), and with cSNVs in at least three cases in total (Supplementary Table 4). We selected cSNVs from each of these 240 genes for re-sequencing to confirm their somatic status. We did not re-sequence genes with previously documented mutations in lymphoma (for example, $C D 79 B, B C L 2$ ). We confirmed the somatic status of $543 \mathrm{cSNVs}$ in 317 genes, with 109 genes having at least two confirmed somatic mutations (Supplementary Tables 4 and $5)$. Of the successfully re-sequenced cSNVs predicted from the genomes, 171 (94.5\%) were confirmed somatic, 7 were false calls and 3 were present in the germ line. These 109 recurrently mutated genes were significantly enriched for genes implicated in lymphocyte activation $\left(P=8.3 \times 10^{-4}\right.$; for example, STAT6, BCL10), lymphocyte differentiation $\left(P=3.5 \times 10^{-3}\right.$; for example, $\left.C A R D 11\right)$, and regulation of apoptosis $\left(P=1.9 \times 10^{-3}\right.$; for example, BTG1, BTG2). Also significantly enriched were genes linked to transcriptional regulation $\left(P=5.4 \times 10^{-4}\right.$; for example, TP53) and genes involved in methylation $\left(P=2.2 \times 10^{-4}\right)$ and acetylation $\left(P=1.2 \times 10^{-2}\right)$, including histone methyltransferase (HMT) and acetyltransferase (HAT) enzymes known previously to be mutated in lymphoma (for example, EZH2 (ref. 13) and CREBBP (ref. 15); Supplementary Methods).

Mutation hot spots can result from mutations at sites under strong selective pressure and we have previously identified such sites using RNA-seq data ${ }^{13}$. We searched our RNA-seq data for genes with mutation hot spots, and identified 10 genes that were not mutated in the 14 genomes (PIM1, FOXO1, CCND3, TP53, IRF4, BTG2, CD79B, $B C L 7 A, I K Z F 3$ and B2M), of which five (FOXO1, CCND3, BTG2,

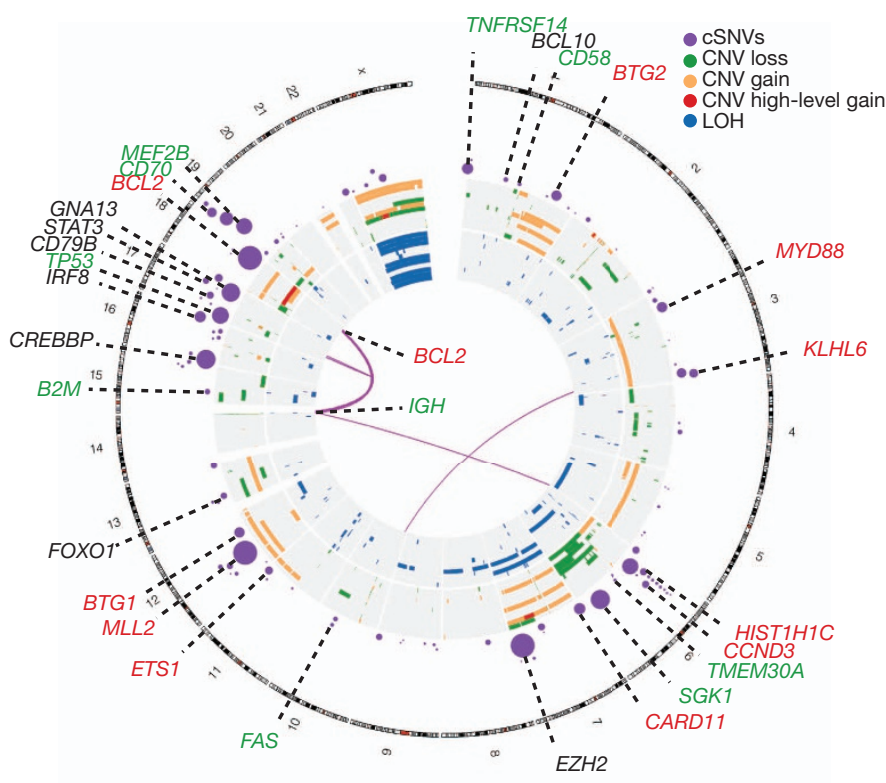

Figure $1 \mid$ Genome-wide visualization of somatic mutation targets in NHL. Overview of structural rearrangements and copy number variations (CNVs) in the 11 DLBCL genomes and cSNVs in the 109 recurrently mutated genes identified in our analysis. Inner arcs represent somatic fusion transcripts identified in at least one of the 11 genomes. The CNVs and LOH detected in each of the 11 DLBCL tumour/normal pairs are displayed on the concentric sets of rings. The inner 11 rings show regions of enhanced homozygosity plotted with blue (interpreted as LOH). The outer 11 rings show somatic CNVs. Purple circles indicate the position of genes with at least two confirmed somatic mutations with circle diameter proportional to the number of cases with cSNVs detected in that gene. Circles representing the genes with significant evidence for positive selection are labelled. Coincidence between recurrently mutated genes and regions of gain/loss are colour-coded in the labels (green, loss; red, gain). For example $B 2 M$, which encodes beta-2-microglobulin, is recurrently mutated and is deleted in two cases.
IKZF3 and B2M) were not previously known targets of point mutation in NHL (Supplementary Table 6 and Supplementary Methods). FOXO1, BCL7A and B2M had hot spots affecting their start codons. The effect of a FOXO1 start codon mutation, which was observed in three cases, was further studied using a cell line in which the initiating ATG was mutated to TTG. Western blots probed with a FOXO1 antibody revealed a band with a reduced molecular weight, indicative of a FOXO1 amino-terminal truncation (Supplementary Figure 2), consistent with use of the next in-frame ATG for translation initiation. A second hot spot in FOXO1 at T24 was mutated in two cases. T24 is reportedly phosphorylated by AKT subsequent to B-cell receptor (BCR) stimulation ${ }^{16}$ inducing FOXO1 nuclear export.

We analysed the RNA-seq data to determine whether any of the somatic mutations in the 109 recurrently mutated genes showed evidence for allelic imbalance with expression favouring one allele. Out of 380 expressed heterozygous mutant alleles, we observed preferential expression of the mutation for $16.8 \%$ (64/380) and preferential expression of the wild type for $27.8 \%$ (106/380; Supplementary Table 7). Seven genes showed evidence for significant preferential expression of the mutant allele in at least two cases: BCL2, CARD11, CD79B, EZH2, IRF4, MEF2B and TP53; Supplementary Methods. In 27 out of 43 cases with BCL 2 cSNVs, expression favoured the mutant allele, consistent with the previously-described hypothesis that the translocated (and hence, transcriptionally deregulated) allele of $B C L 2$ is targeted by somatic hypermutation ${ }^{17}$. Examples of mutations at known oncogenic hot spot sites such as F123I in CARD11 (ref. 18) showed allelic imbalance favouring the mutant allele in some cases. Similarly, we noted expression favouring two novel hot spot mutations in MEF2B (Y69 and D83) and two sites in EZH2 not previously reported as mutated in lymphoma (A682G and A692V).

We sought to distinguish new cancer-related mutations from passenger mutations using the approach proposed previously ${ }^{19}$. We reasoned that this would reveal genes with strong selection signatures, and mutations in such genes would be good candidate cancer drivers. We identified 26 genes with significant evidence for positive selection (false discovery rate $=0.03$, Supplementary Methods), with either selective pressure for acquiring non-synonymous point mutations or truncating/nonsense mutations (Supplementary Methods; Table 1 and Supplementary Table 8). Included were known lymphoma oncogenes (BCL2, CD79B (ref. 9), CARD11 (ref. 18), MYD88 (ref. 10) and EZH2 (ref. 13)), all of which showed signatures indicative of selection for non-synonymous variants.

\section{Evidence for selection of inactivating changes}

We expected tumour suppressor genes to show strong selection for the acquisition of nonsense mutations. In our analysis, the eight most significant genes included seven with strong selective pressure for nonsense mutations, including the known tumour suppressor genes TP53 and TNFRSF14 (ref. 20 ; Table 1). CREBBP, recently reported as commonly inactivated in DLBCL ${ }^{15}$, also showed some evidence for acquisition of nonsense mutations and cSNVs (Supplementary Figure 3 and Supplementary Table 9). We also observed enrichment for nonsense mutations in BCL10, a positive regulator of $N F-\kappa B$, in which oncogenic truncated products have been described in lymphomas ${ }^{21}$. The remaining strongly significant genes (BTG1, GNA13, SGK1 and $M L L 2)$ had no reported role in lymphoma. GNA13 was affected by mutations in 22 cases including multiple nonsense mutations. GNA13 encodes the alpha subunit of a heterotrimeric G-protein coupled receptor responsible for modulating RhoA activity ${ }^{22}$. Some of the mutated residues negatively affect its function ${ }^{23,24}$, including a T203A mutation, which also showed allelic imbalance favouring the mutant allele (Supplementary Table 7). GNA13 protein was reduced or absent on western blots in cell lines harbouring either a nonsense mutation, a stop codon deletion, a frame shifting deletion, or changes affecting splice sites (Supplementary Methods and Supplementary Figure 4). 
Table 1 | Overview of cSNVs and confirmed somatic mutations in most frequently mutated genes

\begin{tabular}{|c|c|c|c|c|c|c|c|c|c|c|c|c|}
\hline \multirow[t]{2}{*}{ Gene } & \multicolumn{3}{|c|}{ Cases } & \multicolumn{3}{|c|}{ Total } & \multirow{2}{*}{$\begin{array}{c}\text { Somatic cSNVs } \\
\text { (RNA-seq } \\
\text { cohort)* }\end{array}$} & \multirow[t]{2}{*}{$P$ (raw) } & \multirow[t]{2}{*}{$q$} & \multirow[t]{2}{*}{ NS SP } & \multirow[t]{2}{*}{ T SP } & \multirow{2}{*}{$\begin{array}{c}\text { Skew } \\
\text { (M, WT, both) }\end{array}$} \\
\hline & NS & S & $T$ & NS & S & $T$ & & & & & & \\
\hline MLL2: & 16 & 8 & 17 & 17 & 8 & 18 & 10 & $6.85 \times 10^{-8}$ & $8.50 \times 10^{-7}$ & 0.834 & 14.4 & WT \\
\hline TNFRSF14 ${ }^{G_{*}}$ & 7 & 1 & 7 & 8 & 1 & 7 & 11 & $6.85 \times 10^{-8}$ & $8.50 \times 10^{-7}$ & 7.52 & 118 & Both \\
\hline$S G K 1^{G_{*}}$ & 18 & 6 & 6 & 37 & 10 & 6 & 9 & $6.85 \times 10^{-8}$ & $8.50 \times 10^{-7}$ & 19.5 & 61.7 & - \\
\hline BCL10\% & 2 & 0 & 4 & 3 & 0 & 4 & 4 & $6.85 \times 10^{-8}$ & $8.50 \times 10^{-7}$ & 3.62 & 112 & WT \\
\hline GNA13 ${ }^{G_{+}}$ & 21 & 1 & 2 & 33 & 1 & 2 & 5 & $6.85 \times 10^{-8}$ & $8.50 \times 10^{-7}$ & 24.1 & 25.7 & Both \\
\hline TP53 $G_{+}$ & 20 & 2 & 1 & 23 & 3 & 1 & 22 & $6.85 \times 10^{-8}$ & $8.50 \times 10^{-7}$ & 15.6 & 14.1 & Both \\
\hline$E Z H 2 G_{*}^{*}$ & 33 & 0 & 0 & 33 & 0 & 0 & 33 & $6.85 \times 10^{-8}$ & $8.50 \times 10^{-7}$ & 11.4 & 0.00 & Both \\
\hline BTG2: & 12 & 6 & 1 & 14 & 6 & 1 & 2 & $6.85 \times 10^{-8}$ & $8.50 \times 10^{-7}$ & 23.9 & 35.1 & - \\
\hline$B C L 2 \mathrm{G}_{*}$ & 42 & 45 & 0 & 96 & 105 & 0 & 43 & $9.35 \times 10^{-8}$ & $8.50 \times 10^{-7}$ & 3.78 & 0.00 & M \\
\hline$B C L 6 \# \S$ & 11 & 2 & 0 & 12 & 2 & 0 & 2 & $9.35 \times 10^{-8}$ & $8.50 \times 10^{-7}$ & 0.175 & 0.00 & M \\
\hline CIITA† & 5 & 3 & 0 & 6 & 3 & 0 & 2 & $9.35 \times 10^{-8}$ & $8.50 \times 10^{-7}$ & 0.086 & 0.00 & \\
\hline FAS: & 2 & 0 & 4 & 3 & 0 & 4 & 2 & $1.52 \times 10^{-7}$ & $1.17 \times 10^{-6}$ & 2.54 & 66.5 & WT \\
\hline BTG1末 & $1 \overline{1}$ & 6 & 2 & 11 & 7 & 2 & 10 & $1.52 \times 10^{-7}$ & $1.17 \times 10^{-6}$ & 17.5 & 52.5 & Both \\
\hline$M E F 2 B^{G}+$ & 20 & 2 & 0 & 20 & 2 & 0 & 10 & $2.05 \times 10^{-7}$ & $1.47 \times 10^{-6}$ & 14.2 & 0.00 & $\mathrm{M}$ \\
\hline IRF8: & 11 & 5 & 3 & 14 & 5 & 3 & 3 & $4.55 \times 10^{-7}$ & $3.03 \times 10^{-6}$ & 8.82 & 28.2 & WT \\
\hline TMEMЗОA: & 1 & 0 & 4 & 1 & 0 & 4 & 4 & $6.06 \times 10^{-7}$ & $3.79 \times 10^{-6}$ & 0.785 & 65.0 & WT \\
\hline CD58: & 2 & 0 & 3 & 2 & 0 & 3 & 2 & $2.42 \times 10^{-6}$ & $1.43 \times 10^{-5}$ & 2.29 & 69.2 & - \\
\hline KLHL6: & 10 & 2 & 2 & 12 & 2 & 2 & 4 & $1.00 \times 10^{-5}$ & $5.26 \times 10^{-5}$ & 5.42 & 16.4 & - \\
\hline MYD88 ${ }^{\mathrm{A}}+$ & 13 & 2 & 0 & 14 & 2 & 0 & 9 & $1.00 \times 10^{-5}$ & $5.26 \times 10^{-5}$ & 12.4 & 0.00 & WT \\
\hline CD70 & 5 & 0 & 1 & 5 & 0 & 2 & 3 & $1.70 \times 10^{-5}$ & $8.48 \times 10^{-5}$ & 7.08 & 44.0 & - \\
\hline$C D 79 B^{A_{+}}$ & 7 & 2 & 1 & 9 & 2 & 1 & 5 & $2.00 \times 10^{-5}$ & $9.52 \times 10^{-5}$ & 10.9 & 18.3 & $\mathrm{M}$ \\
\hline CCND3 $\ddagger^{\circ}$ & 7 & 1 & 2 & 7 & 1 & 2 & 6 & $2.80 \times 10^{-5}$ & $1.27 \times 10^{-4}$ & 6.55 & 36.3 & WT \\
\hline CREBBP: & 20 & 7 & 4 & 24 & 7 & 4 & 9 & $1.00 \times 10^{-4}$ & $4.35 \times 10^{-4}$ & 2.72 & 6.04 & Both \\
\hline HISTIHIC. & 9 & 0 & 0 & 10 & 0 & 0 & 6 & $1.80 \times 10^{-4}$ & $7.50 \times 10^{-4}$ & 11.9 & 0.00 & Both \\
\hline$B 2 M *$ & 7 & 0 & 0 & 7 & 0 & 0 & 4 & $3.90 \times 10^{-4}$ & $1.56 \times 10^{-3}$ & 16.6 & 0.00 & WT \\
\hline ETS1 & 10 & 1 & 0 & 10 & 1 & 0 & 4 & $4.10 \times 10^{-4}$ & $1.58 \times 10^{-3}$ & 5.76 & 0.00 & WT \\
\hline CARD11† & 14 & 3 & 0 & 14 & 3 & 0 & 3 & $1.90 \times 10^{-3}$ & $7.04 \times 10^{-3}$ & 3.37 & 0.00 & Both \\
\hline FAT2† & 2 & 1 & 0 & 2 & 1 & 0 & 2 & $6.30 \times 10^{-3}$ & $2.25 \times 10^{-2}$ & 0.128 & 0.00 & - \\
\hline IRF4†§ & 9 & 4 & 0 & 26 & 5 & 0 & 5 & $7.00 \times 10^{-3}$ & $2.41 \times 10^{-2}$ & 0.569 & 0.00 & Both \\
\hline FOXO1: & 8 & 4 & 0 & 10 & 4 & 0 & 4 & $7.60 \times 10^{3}$ & $2.53 \times 10^{-2}$ & 4.02 & 0.00 & - \\
\hline STAT3 & 9 & 0 & 0 & 9 & 0 & 0 & 4 & $2.19 \times 10^{-2}$ & $6.08 \times 10^{-2}$ & - & - & Both \\
\hline RAPGEF1 & 8 & 3 & 0 & 10 & 3 & 0 & 3 & $2.98 \times 10^{-2}$ & $7.45 \times 10^{-2}$ & - & - & WT \\
\hline$A B C A 7$ & 12 & 3 & 0 & 15 & 3 & 0 & 2 & $7.76 \times 10^{-2}$ & $1.67 \times 10^{-1}$ & - & - & WT \\
\hline RNF213 & 10 & 8 & 0 & 10 & 8 & 0 & 2 & $7.87 \times 10^{-2}$ & $1.67 \times 10^{-1}$ & - & - & - \\
\hline MUC16 & 17 & 12 & 0 & 39 & 25 & 0 & 2 & $8.32 \times 10^{-2}$ & $1.73 \times 10^{-1}$ & - & - & - \\
\hline HDAC7 & 8 & 4 & 0 & 8 & 4 & 0 & 2 & $8.94 \times 10^{-2}$ & $1.82 \times 10^{-1}$ & - & - & WT \\
\hline$P R K D C$ & 7 & 3 & 0 & 7 & 4 & 0 & 2 & $1.06 \times 10^{-1}$ & $2.05 \times 10^{-1}$ & - & - & - \\
\hline SAMD9 & 9 & 2 & 0 & 9 & 2 & 0 & 2 & $1.79 \times 10^{-1}$ & $3.01 \times 10^{-1}$ & - & - & - \\
\hline TAF1 & 10 & 0 & 0 & 10 & 0 & 0 & 2 & $3.03 \times 10^{-1}$ & $4.74 \times 10^{-1}$ & - & - & - \\
\hline PIM1 & 20 & 19 & 0 & 33 & 34 & 0 & 11 & $3.40 \times 10^{-1}$ & $5.23 \times 10^{-1}$ & - & - & WT \\
\hline COL $4 A 2$ & 8 & 2 & 0 & 8 & 2 & 0 & 2 & $7.64 \times 10^{-1}$ & $8.99 \times 10^{-1}$ & - & - & - \\
\hline EP300 & 8 & 7 & 1 & 8 & 7 & 1 & 3 & $9.54 \times 10^{-1}$ & 1.00 & - & - & WT \\
\hline
\end{tabular}

Individual cases with non-synonymous (NS), synonymous (S) and truncating (T) mutations and the total number of mutations of each class are shown separately because some genes contained multiple

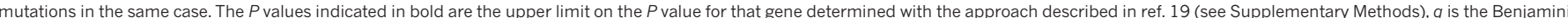

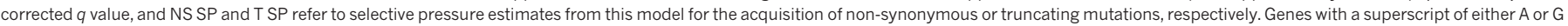
were found to have mutations significantly enriched in $A B C$ or GCB cases, respectively $(P<0.05$, Fisher's exact test).

* Additional somatic mutations identified in larger cohorts and insertion/deletion mutations are not included in this total.

† 'Both' indicates that we observed separate cases in which skewed expression was seen but where this skew was not consistent for the mutant or wild-type allele.

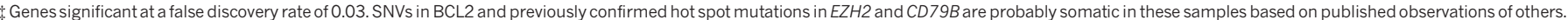
$\S$ Selective pressure estimates are both $<1$ indicating purifying selection rather than positive selection acting on this gene.

SGK1 encodes a phosphatidylinositol-3-OH kinase (PI(3)K)regulated kinase with functions including regulation of FOXO transcription factors ${ }^{25}$, regulation of NF- $\kappa \mathrm{B}$ by phosphorylating $\mathrm{I} \kappa \mathrm{B}$ kinase $^{26}$, and negative regulation of NOTCH signalling ${ }^{27}$. SGK1 also resides within a region of chromosome 6 commonly deleted in DLBCL (Fig. 1$)^{5}$. The mechanism by which SGK1 and GNA13 inactivation may contribute to lymphoma is unclear, but the strong degree of apparent selection towards their inactivation and their overall high mutation frequency (each mutated in 18 of 106 DLBCL cases) suggests that their loss contributes to B-cell NHL. Certain genes are known to be mutated more commonly in GCB DLBCLs (for example, TP53 (ref. 28) and EZH2 (ref. 13)). Here, both SGK1 and GNA13 mutations were found only in GCB cases $\left(P=1.93 \times 10^{-3}\right.$ and $2.28 \times 10^{-4}$, Fisher's exact test; $n=15$ and 18, respectively) (Fig. 2). Two additional genes (MEF2B and TNFRSF14) with no previously described role in DLBCL showed a similar restriction to GCB cases (Fig. 2).

\section{Inactivating MLL2 mutations}

MLL2 showed the most significant evidence for selection and the largest number of nonsense SNVs. Our RNA-seq analysis indicated that $26.0 \%(33 / 127)$ of cases carried at least one MLL2 cSNV. To address the possibility that variable RNA-seq coverage of $M L L 2$ failed to capture some mutations, we PCR-amplified the entire MLL2 locus ( $\sim 36$ kilobases) in 89 cases ( 35 primary FLs, 17 DLBCL cell lines, and 37 DLBCLs). Of these cases 58 were among the RNA-seq cohort. Illumina amplicon re-sequencing (Supplementary Methods) revealed 78 mutations, confirming the RNA-seq mutations in the overlapping cases and identifying 33 additional mutations. We confirmed the somatic status of 46 variants using Sanger sequencing (Supplementary Table 10), and showed that 20 of the 33 additional mutations were insertions or deletions (indels). Three SNVs at splice sites were also detected, as were 10 new cSNVs that had not been detected by RNA-seq.

The somatic mutations were distributed across MLL2 (Fig. 3a). Of these, $37 \%(n=29 / 78)$ were nonsense mutations, $46 \%(n=36 / 78)$ were indels that altered the reading frame, $8 \%(n=6 / 78)$ were point mutations at splice sites and $9 \%(n=7 / 78)$ were non-synonymous amino acid substitutions (Table 2). Four of the somatic splice site mutations had effects on MLL2 transcript length and structure. For example, two heterozygous splice site mutations resulted in the use of a novel splice donor site and an intron retention event.

Approximately half of the NHL cases we sequenced had two MLL2 mutations (Supplementary Table 10). We used bacterial artificial 


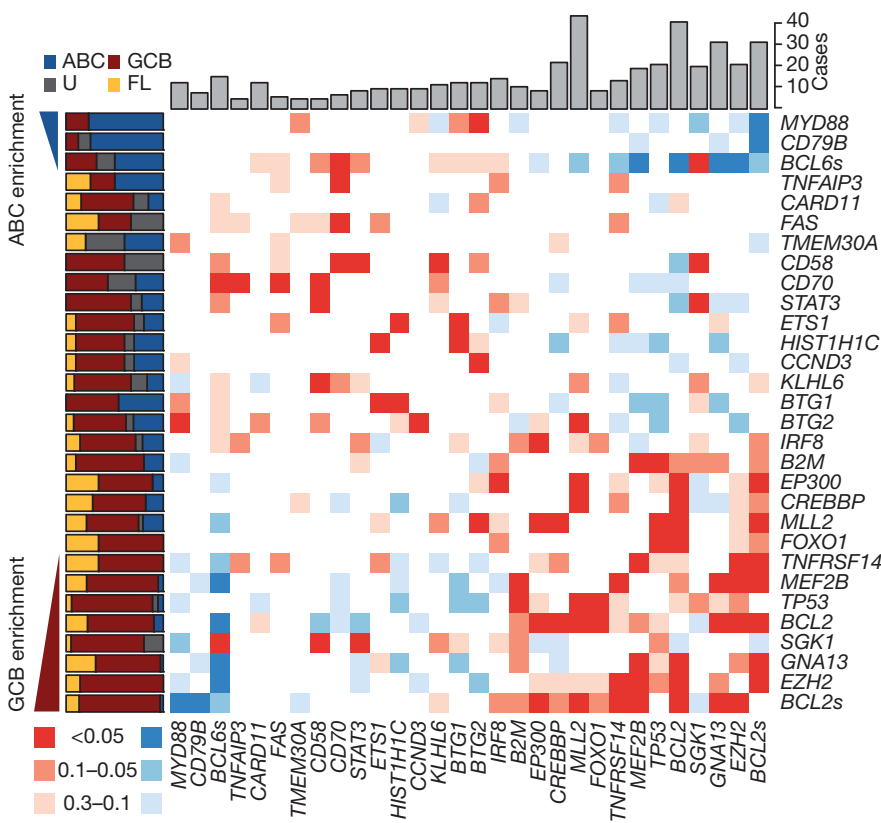

Figure $2 \mid$ Overview of mutations and potential cooperative interactions in NHL. This heat map displays possible trends towards co-occurrence (red) and mutual exclusion (blue) of somatic mutations and structural rearrangements. Colours were assigned by taking the minimum value of a left- and right-tailed Fisher's exact test. To capture trends a $P$-value threshold of 0.3 was used, with the darkest shade of the colour indicating those meeting statistical significance $(P \leq 0.05)$. The relative frequency of mutations in ABC (blue), GCB (red), unclassifiable (black) DLBCLs and FL (yellow) cases is shown on the left. Genes were arranged with those having significant $(P<0.05$, Fisher's exact test) enrichment for mutations in $\mathrm{ABC}$ cases (blue triangle) towards the top (and left) and those with significant enrichment for mutations in GCB cases (red triangle) towards the bottom (and right). The total number of cases in which each gene contained either cSNVs or confirmed somatic mutations is shown at the top. The cluster of blue squares (upper-right) results from the mutual exclusion of the ABC-enriched mutations (for example, MYD88, CD79B) from the GCB-enriched mutations (for example, EZH2, GNA13). Presence of structural rearrangements involving the two oncogenes BCL6 and BCL2 (indicated as BCL6s and BCL2s) was determined with FISH techniques using break-apart probes (Supplementary Methods).

chromosome (BAC) clone sequencing in eight FL cases to show that in all eight cases the mutations were in trans, affecting both MLL2 alleles. This observation is consistent with the notion that there is a complete, or near-complete, loss of MLL2 in the tumour cells of such patients.

With the exception of two primary FL cases and two DLBCL cell lines (Pfeiffer and SU-DHL-9), the majority of MLL2 mutations seemed to be heterozygous. Analysis of Affymetrix 500k SNP array data from two FL cases with apparent homozygous mutations revealed that both tumours showed copy number neutral loss of heterozygosity (LOH) for the region of chromosome 12 containing MLL2 (Supplementary Methods). Thus, in addition to bi-allelic mutation, $\mathrm{LOH}$ is a second, albeit less common mechanism by which MLL2 function is lost.

MLL2 was the most frequently mutated gene in FL, and among the most frequently mutated genes in DLBCL (Fig. 2). We confirmed MLL2 mutations in 31 of $35 \mathrm{FL}$ patients $(89 \%)$, in 12 of 37 DLBCL patients (32\%), in 10 of 17 DLBCL cell lines (59\%) and in none of the eight normal centroblast samples we sequenced. Our analysis predicted that the majority of the somatic mutations observed in MLL2 were inactivating ( $91 \%$ disrupted the reading frame or were truncating point mutations), indicating to us that MLL2 is a tumour suppressor of significance in NHL.

\section{Recurrent point mutations in MEF2B}

Our selective pressure analysis also revealed genes with stronger pressure for acquisition of amino acid substitutions than for nonsense

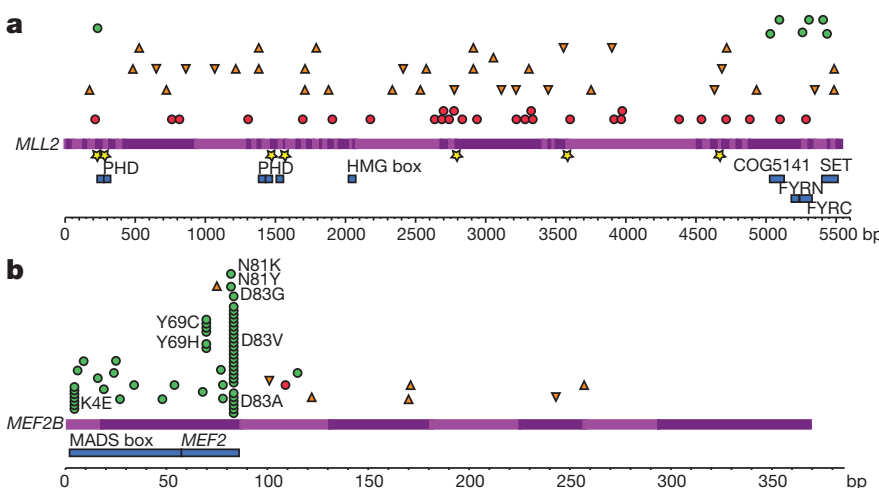

Figure 3 Summary and effect of somatic mutations affecting $M L L 2$ and MEF2B. a, Re-sequencing the MLL2 locus in 89 samples revealed mainly nonsense (red circles) and frameshift-inducing indel mutations (orange triangles; inverted triangles for insertions and upright triangles for deletions). A smaller number of non-synonymous somatic mutations (green circles) and point mutations or deletions affecting splice sites (yellow stars) were also observed. All of the non-synonymous point mutations affected a residue within either the catalytic SET domain, the FYRC domain (FY-rich carboxy-terminal domain) or PHD zinc finger domains. The effect of these splice-site mutations on MLL2 splicing was also explored (Supplementary Figure 7). b. The cSNVs and somatic mutations found in $M E F 2 B$ in all FL and DLBCL cases sequenced are shown with the same symbols. Only the amino acids with variants in at least two patients are labelled. cSNVs were most prevalent in the first two proteincoding exons of MEF2B (exons 2 and 3). The crystal structure of MEF2 bound to EP300 supports the idea that two of the mutated sites (L67 and Y69) are important in the interaction between these proteins (Supplementary Figure 8 and Supplementary Discussion) $)^{50}$.

mutations. One such gene was $M E F 2 B$, which had not previously been linked to lymphoma. We found that $20(15.7 \%)$ cases had MEF2B cSNVs and $4(3.1 \%)$ cases had MEF2C cSNVs. All cSNVs detected by RNA-seq affected either the MADS box or MEF2 domains. To determine the frequency and scope of $M E F 2 B$ mutations, we Sangersequenced exons 2 and 3 in 261 primary FL samples; 259 DLBCL primary tumours; 17 cell lines; 35 cases of assorted NHL (IBL, composite FL and PBMCL); and eight non-malignant centroblast samples. We also used a capture strategy (Supplementary Methods) to sequence the entire $M E F 2 B$ coding region in the $261 \mathrm{FL}$ samples, revealing six additional variants outside exons 2 and 3. We thus identified 69 cases (34 DLBCL, 12.67\%; and 35 FL, 15.33\%) with MEF2B cSNVs or indels, failing to observe novel variants in other NHL and non-malignant samples. Of the variants 55 (80\%) affected residues within the MADS box and MEF2 domains encoded by exons 2 and 3 (Supplementary Table 11; Fig. 3b). Each patient generally had a single $M E F 2 B$ variant and we observed relatively few (eight in total, 10.7\%) truncation-inducing SNVs or indels. Non-synonymous SNVs were by far the most common type of change observed, with $59.4 \%$ of detected variants affecting K4, Y69, N81 or D83. In 12 cases $M E F 2 B$ mutations were shown to be somatic, including representative mutations at each of K4, Y69, N81 and D83 (Supplementary Table 12). We did not detect mutations in $\mathrm{ABC}$ cases, indicating that somatic mutations in $M E F 2 B$ have a role unique to the development of GCB DLBCL and FL (Fig. 2).

Table 2 | Summary of types of MLL2 somatic mutations

\begin{tabular}{lcccc}
\hline Sample Type & FL & DLBCL & DLBCL cell-line & Centroblast \\
\hline Truncation & 18 & 4 & 7 & 0 \\
Indel with frameshift & 22 & 8 & 6 & 0 \\
Splice site & 4 & 2 & 0 & 0 \\
SNV & 3 & 2 & 2 & 0 \\
Any mutation/ & & & & \\
number of cases & $31 / 35$ & $12 / 37$ & $10 / 17$ & $0 / 8$ \\
Percentage & 89 & 32 & 59 & 0 \\
\hline
\end{tabular}




\section{Discussion}

In our study of genome, transcriptome and exome sequences from 127 B-cell NHL cases, we identified 109 genes with clear evidence of somatic mutation in multiple individuals. Significant selection seems to act on at least 26 of these for the acquisition of either nonsense or missense mutations. To the best of our knowledge, the majority of these genes had not previously been associated with any cancer type. We observed an enrichment of somatic mutations affecting genes involved in transcriptional regulation and, more specifically, chromatin modification.

MLL2 emerged from our analysis as a major tumour suppressor locus in NHL. It is one of six human H3K4-specific methyltransferases $^{29}$, all of which share homology with the Drosophila trithorax gene. Trimethylated $\mathrm{H} 3 \mathrm{~K} 4(\mathrm{H} 3 \mathrm{~K} 4 \mathrm{me} 3)$ is an epigenetic mark associated with the promoters of actively transcribed genes. By laying down this mark, MLLs are responsible for the transcriptional regulation of developmental genes including the homeobox (Hox) gene family ${ }^{30}$ which collectively control segment specificity and cell fate in the developing embryo ${ }^{31,32}$. Each MLL family member is thought to target different subsets of Hox genes ${ }^{33}$ and in addition, MLL2 is known to regulate the transcription of a diverse set of genes ${ }^{34}$. Recently, MLL2 mutations were reported in a small-cell lung cancer cell line $\mathrm{e}^{35}$ and in renal carcinoma ${ }^{36}$, but the frequency of nonsense mutations affecting $M L L 2$ in these cancers was not established in these reports. Inactivating mutations were reported recently in MLL2 or MLL3 in $16 \%$ of medulloblastoma patients ${ }^{37}$, further implicating $M L L 2$ as a cancer gene.

Our data link MLL2 somatic mutations to B-cell NHL. The reported mutations are likely to be inactivating and in eight of the cases with multiple mutations, we confirmed that both alleles were affected, presumably resulting in essentially complete loss of MLL2 function. The high prevalence of MLL2 mutations in FL (89\%) equals the frequency of the $t(14 ; 18)(\mathrm{q} 32 ; \mathrm{q} 21)$ translocation, which is considered the most prevalent genetic abnormality in $\mathrm{FL}^{3}$. In DLBCL tumour samples and cell lines, MLL2 mutation frequencies were $32 \%$ and $59 \%$, respectively, also exceeding the prevalence of the most frequent cytogenetic abnormalities, such as the various translocations involving 3q27, which occur in 25-30\% of DLBCLs and are enriched in $\mathrm{ABC}$ cases $^{38}$. Importantly, we found MLL2 mutated in both DLBCL subtypes (Fig. 2). Our analyses thus indicate that MLL2 acts as a central tumour suppressor in FL and both DLBCL subtypes.

The MEF2 gene family encodes four related transcription factors that recruit histone-modifying enzymes including histone deacetylases (HDACs) and HATs in a calcium-regulated manner. Although truncating variants were detected in our analysis of $M E F 2$ gene family members, our analysis suggests that, in contrast to $M L L 2, M E F 2$ family members tend to selectively acquire non-synonymous amino acid substitutions. In the case of $M E F 2 B, 59.4 \%$ of all the $\mathrm{CSNV}$ s were found at four sites within the protein (K4, Y69, N81 and D83), and all four of these sites were confirmed to be targets of somatic mutation. D83 is affected in $39 \%$ of the $M E F 2 B$ alterations, resulting in replacement of the charged aspartate with any of alanine, glycine or valine. Although we cannot yet predict the consequences of these substitutions on protein function, it seems likely that their effect would have an impact on the ability of MEF2B to facilitate gene expression and thus have a role in promoting the malignant transformation of germinal centre $B$ cells to lymphoma (Supplementary Discussion).

$M E F 2 B$ mutations can be linked to CREBBP and EP300 mutations, and to recurrent Y641 mutations in EZH2 (ref. 13). One target of CREBBP/EP300 HAT activity is $\mathrm{H} 3 \mathrm{~K} 27$, which is methylated by $\mathrm{EZH} 2$ to repress transcription. There is evidence that the action of EZH2 antagonizes that of CREBBP/EP300 (ref. 39). One function of MEF2 is to recruit either HDACs or CREBBP/EP300 to target genes ${ }^{40}$, and it has been suggested that HDACs compete with CREBBP/EP300 for the same binding site on MEF2 (ref. 41). Under normal $\mathrm{Ca}^{2+}$ levels, MEF2 is bound by type IIa HDACs, which maintain the tails of histone proteins in a deacetylated repressive chromatin state ${ }^{42}$ Increased cytoplasmic $\mathrm{Ca}^{2+}$ levels induce the nuclear export of HDACs, enabling the recruitment of HATs such as CREBBP/ EP300, facilitating transcription at MEF2 target genes. Mutation of $C R E B B P, E P 300$ or MEF2B may have an impact on the expression of MEF2 target genes owing to reduced acetylation of nucleosomes near these genes (Supplementary Figure 5; Supplementary Discussion). In light of the recent finding that heterozygous EZH2 Y641 mutations enhance overall H3K27 trimethylation activity of PRC2 (refs 43, 44), it is possible that mutation of both MLL2 and EZH2 could cooperate in reducing the expression of some of the same target genes. Our data indicate that (1) post-transcriptional modification of histones is of key importance in germinal centre B cells and (2) deregulated histone modification due to these mutations is likely to result in reduced acetylation and enhanced methylation, and acts as a core driver event in the development of NHL (Supplementary Figure 5).

\section{METHODS SUMMARY}

All samples analysed contained at least 50\% tumour cells. Genomes, exomes and transcriptomes were sequenced using a combination of Illumina GAIIx and HiSeq 2000 instruments to read lengths of between 36 and 100 nucleotides. Exome capture was performed using the Agilent SureSelect Target Enrichment System Protocol (Version 1.0, September 2009). Alignment was accomplished using BWA ${ }^{45}$ and variants were identified using $\mathrm{SNVmix}^{46}$. Variants were manually reviewed in IGV and were confirmed (where applicable) by PCR followed by either Sanger sequencing or Illumina re-sequencing. Structural rearrangements in genomes and transcriptomes were identified using $\mathrm{ABySS}^{47}$. Gene expression values used for subtype assignment were calculated as reads per kilobase gene model per million mapped reads (RPKM) values ${ }^{48}$ and subtypes were assigned using an adaptation of the method developed for data from Affymetrix expression arrays $^{49}$ trained with samples previously classified by this standard approach.

\section{Received 13 November 2010; accepted 7 July 2011.}

\section{Published online 27 July 2011.}

1. Anderson, J. R., Armitage, J. O., Weisenburger, D. D., Non-Hodgkin's Lymphoma Classification Project. Epidemiology of the non-Hodgkin's lymphomas: distributions of the major subtypes differ by geographic locations. Ann. Oncol. 9, 717-720 (1998).

2. Lenz, G. \& Staudt, L. M. Aggressive Iymphomas. N. Engl. J. Med. 362, 1417-1429 (2010).

3. Horsman, D. E. et al. Follicular lymphoma lacking the $t(14 ; 18)(q 32 ; q 21)$ : identification of two disease subtypes. Br. J. Haematol. 120, 424-433 (2003).

4. Iqbal, J. et al. BCL2 translocation defines a unique tumor subset within the germinal center B-cell-like diffuse large B-cell lymphoma. Am. J. Pathol. 165, 159-166 (2004).

5. Lenz, G. et al. Molecular subtypes of diffuse large B-cell lymphoma arise by distinct genetic pathways. Proc. Natl Acad. Sci. USA 105, 13520-13525 (2008).

6. Pasqualucci, L. et al. Inactivation of the PRDM1/BLIMP1 gene in diffuse large B cell lymphoma. J. Exp. Med. 203, 311-317 (2006).

7. Kato, M. et al. Frequent inactivation of A20 in B-cell lymphomas. Nature 459, 712-716 (2009).

8. Compagno, M. et al. Mutations of multiple genes cause deregulation of NF- $\mathrm{KB}$ in diffuse large B-cell lymphoma. Nature 459, 717-721 (2009).

9. Davis, R. E. et al. Chronic active B-cell-receptor signalling in diffuse large B-cell lymphoma. Nature 463, 88-92 (2010).

10. Ngo, V. N. et al. Oncogenically active MYD 88 mutations in human lymphoma. Nature 470, 115-119 (2011).

11. Mardis, E. R. et al. Recurring mutations found by sequencing an acute myeloid leukemia genome. N. Engl. J. Med. 361, 1058-1066 (2009).

12. Shah, S. P. et al. Mutational evolution in a lobular breast tumour profiled at single nucleotide resolution. Nature 461, 809-813 (2009).

13. Morin, R. D. et al. Somatic mutations altering EZH2 (Tyr641) in follicular and diffuse large B-cell lymphomas of germinal-center origin. Nature Genet. $\mathbf{4 2}$, 181-185 (2010).

14. Futreal, P. A. etal. A census of human cancer genes. Nature Rev. Cancer 4, 177-183 (2004).

15. Pasqualucci, L. et al. Inactivating mutations of acetyltransferase genes in B-cell lymphoma. Nature 471, 189-195 (2011).

16. Yusuf, I., Zhu, X., Kharas, M. G., Chen, J. \& Fruman, D. A. Optimal B-cell proliferation requires phosphoinositide 3-kinase-dependent inactivation of FOXO transcription factors. Blood 104, 784-787 (2004).

17. Saito, M. et al. BCL6 suppression of BCL2 via Miz1 and its disruption in diffuse large B cell lymphoma. Proc. Natl Acad. Sci. USA 106, 11294-11299 (2009).

18. Lenz, G. et al. Oncogenic CARD11 mutations in human diffuse large B cell lymphoma. Science 319, 1676-1679 (2008). 
19. Greenman, C. Wooster, R, Futreal, P. A. Stratton, M. R. \& Easton, D. F. Statistical analysis of pathogenicity of somatic mutations in cancer. Genetics 173 , 2187-2198 (2006)

20. Cheung, K. J. et al. Acquired TNFRSF14 mutations in follicular lymphoma are associated with worse prognosis. Cancer Res. 70, 9166-9174 (2010).

21. Du, M. Q. et al. BCL10 gene mutation in lymphoma. Blood 95, 3885-3890 (2000).

22. Kreutz, B., Hajicek, N.., Yau, D. M., Nakamura, S. \& Kozasa, T. Distinct regions of Ga13 participate in its regulatory interactions with RGS homology domaincontaining RhoGEFs. Cell. Signal. 19, 1681-1689 (2007).

23. Bhattacharyya, R. \& Wedegaertner, P. G 13 requires palmitoylation for plasma membrane localization, Rho-dependent signaling, and promotion of p115RhoGEF membrane binding. J. Biol. Chem. 275, 14992-14999 (2000).

24. Manganello, J. M., Huang, J., Kozasa, T., Voyno-Yasenetskaya, T. A. \& Le Breton, G. C. Protein kinase A-mediated phosphorylation of the $\mathrm{G} \alpha 13$ switch I region alters the G $\alpha \beta \gamma 13-G$ protein-coupled receptor complex and inhibits Rho activation. J. Biol. Chem. 278, 124-130 (2003).

25. Brunet, A. et al. Protein kinase SGK mediates survival signals by phosphorylating the forkhead transcription factor FKHRL1 (FOXO3a). Mol. Cell. Biol. 21, 952-965 (2001).

26. Tai, D. J. C. Su, C.-C., Ma, Y.-L. \& Lee, E. H. Y. SGK1 phosphorylation of IкB kinase a and p300 Up-regulates NF-kB activity and increases $\mathrm{N}$-methyl-D-aspartate receptor NR2A and NR2B expression. J. Biol. Chem. 284, 4073-4089 (2009).

27. Mo, J. et al. Serum- and glucocorticoid-inducible kinase 1 (SGK1) controls Notch1 signaling by downregulation of protein stability through Fbw7 ubiquitin ligase. J. Cell Sci. 124, 100-112 (2011).

28. Young, K. H. et al. Structural profiles of TP53 gene mutations predict clinical outcome in diffuse large B-cell lymphoma: an international collaborative study. Blood 112, 3088-3098 (2008).

29. Shilatifard, A. Molecular implementation and physiological roles for histone $\mathrm{H} 3$ lysine 4 (H3K4) methylation. Curr. Opin. Cell Biol. 20, 341-348 (2008)

30. Milne, T. et al. MLL targets SET domain methyltransferase activity to Hox gene promoters. Mol. Cell 10, 1107-1117 (2002).

31. Krumlauf, R. Hox genes in vertebrate development. Cell 78, 191-201 (1994).

32. Canaani, E. et al. ALL-1//MLL1, a homologue of Drosophila TRITHORAX, modifies chromatin and is directly involved in infant acute leukaemia. Br. J. Cancer 90 756-760 (2004).

33. Wang, P. et al. Global analysis of H3K4 methylation defines MLL family member targets and points to a role for MLL1-mediated H3K4 methylation in the regulation of transcriptional initiation by RNA polymerase II. Mol. Cell. Biol. 29, 6074-6085 (2009).

34. Issaeva, I. et al. Knockdown of ALR (MLL2) reveals ALR target genes and leads to alterations in cell adhesion and growth. Mol. Cell. Biol. 27, 1889-1903 (2007)

35. Pleasance, E. D. et al. A small-cell lung cancer genome with complex signatures of tobacco exposure. Nature 463, 184-190 (2010).

36. Dalgliesh, G. L. etal. Systematic sequencing of renal carcinoma reveals inactivation of histone modifying genes. Nature 463, 360-363 (2010).

37. Parsons, D. W. et al. The genetic landscape of the childhood cancer medulloblastoma. Science 331, 435-439 (2011).

38. Iqbal, J. et al. Distinctive patterns of BCL6 molecular alterations and their functional consequences in different subgroups of diffuse large B-cell lymphoma. Leukemia 21, 2332-2343 (2007).

39. Pasini, D. et al. Characterization of an antagonistic switch between histone $\mathrm{H} 3$ Iysine 27 methylation and acetylation in the transcriptional regulation of Polycomb group target genes. Nucleic Acids Res. (2010).

40. Giordano, A. \& Avantaggiati, M. p300 and CBP: partners for life and death. J. Cell. Physiol. 181, 218-230 (1999).

41. Han, A., He, J., Wu, Y., Liu, J. O. \& Chen, L. Mechanism of recruitment of class II histone deacetylases by myocyte enhancer factor-2. J. Mol. Biol. 345, 91-102 (2005).

42. Youn, H. \& Liu, J. Cabin 1 represses MEF2-dependent Nur77 expression and T cell apoptosis by controlling association of histone deacetylases and acetylases with MEF2. Immunity 13, 85-94 (2000).

43. Yap, D. B. et al. Somatic mutations at EZH2 Y641 act dominantly through a mechanism of selectively altered PRC2 catalytic activity, to increase H3K27 trimethylation. Blood 117, 2451-2459 (2011).

44. Sneeringer, C. J. et al. Coordinated activities of wild-type plus mutant EZH2 drive tumor-associated hypertrimethylation of lysine 27 on histone H3 (H3K27) in human B-cell lymphomas. Proc. Natl Acad. Sci. USA 107, 20980-20985 (2010).

45. Li, H. \& Durbin, R. Fast and accurate short read alignment with Burrows-Wheeler transform. Bioinformatics 25, 1754-1760 (2009).

46. Goya, R. et al. SNVMix: predicting single nucleotide variants from next-generation sequencing of tumors. Bioinformatics 26, 730-736 (2010).
47. Robertson, G. et al. De novo assembly and analysis of RNA-seq data. Nature Methods 7, 909-912 (2010)

48. Mortazavi, A., Williams, B. A., Mccue, K., Schaeffer, L. \& Wold, B. Mapping and quantifying mammalian transcriptomes by RNA-Seq. Nature Methods 5, 621-628 (2008).

49. Wright, G. et al. A gene expression-based method to diagnose clinically distinct subgroups of diffuse large B cell lymphoma. Proc. Natl Acad. Sci. USA 100, 9991-9996 (2003)

50. He, J. et al. Structure of $\mathrm{p} 300$ bound to MEF2 on DNA reveals a mechanism of enhanceosome assembly. Nucleic Acids Res. (2011).

Supplementary Information is linked to the online version of the paper at www.nature.com/nature.

Acknowledgements This study was funded in part by funding from the National Cancer Institute Office of Cancer Genomics (Contract No. HHSN261200800001E), the Terry Fox Foundation (grant 019001, Biology of Cancer: Insights from Genomic Analyses of Lymphoid Neoplasms) and Genome Canada/Genome British Columbia Grant Competition III (Project Title: High Resolution Analysis of Follicular Lymphoma Genomes) to J.M.C., R.D.G. and M.A.M. We acknowledge support from NIH grants P50CA130805-01 "SPORE in Lymphoma, Tissue Resource Core (PI Fisher)" and 1 U01CA1 14778 "Molecular Signatures to Improve Diagnosis and Outcome in Lymphoma (PIChan)". A.J.M. is a Career Development Program Fellow of the Leukemia and Lymphoma Society. N.A.J. was a research fellow of the Terry Fox Foundation (award NCIC 019005) and the Michael Smith Foundation for Health Research

(ST-PDF-01793). M.A.M. is a Terry Fox Young Investigator and a Michael Smith Senior Research Scholar. R.D.M. is a Vanier Scholar (CIHR) and holds a MSFHR senior graduate studentship. M.M.-L. acknowledges support from a Postdoctoral Fellowship from the Spanish Ministry of Education, under the "Programa Nacional de Movilidad de Recursos Humanos del Plan Nacional de I-D+i 2008-2011". D.W.S. was supported by the Terry Fox Foundation Strategic Health Research Training Program in Cancer Research at Canadian Institutes of Health Research (Grant No. TGT-53912). J.J.S. acknowledges funding from The Canadian Cancer Society and the Canadian Institutes of Health Research. R.G. is supported by a UBC Four Year Fellowship. I.M.M. acknowledges the Canadian Foundation for Innovation for a Leaders Opportunity Fund. The laboratory work for this study was undertaken at the Genome Sciences Centre, British Columbia Cancer Research Centre and the Centre for Translational and Applied Genomics, a program of the Provincial Health Services Authority Laboratories. The authors would like to thank C. Greenman for supplying his software and also acknowledge D. Gerhard and S. Aparicio for discussions and guidance. Special thanks to C. Suragh, R. Roscoe, A. Troussard and A. Drobnies for expert project management assistance, and to the Library Construction, Sequencing and Bioinformatics teams at the Genome Sciences Centre. The content of this publication does not necessarily reflect the views of policies of the Department of Health and Human Services, nor does mention of trade names, commercial products, or organizations imply endorsement by the US Government.

Author Contributions M.A.M., R.D.G., D.E.H., M.H. and J.M.C. conceived of the study and led the design of the experiments. R.D.M. performed the analysis of sequence data, identified mutations and, with M.M.-L.,A.J.M. and M.A.M., produced figures and wrote the manuscript. M.M.-L., A.J.M., D.L.T., S.Chan, S.Chittarajan, D.S., H.M., J.S., M.M., T.Z., A.D., K.T., Y.B., M.R.F., J.T.-W. and T.M.S. designed and performed experiments to amplify, discover and validate mutations. R.G., M.G. and I.M.M. contributed to analyses and reviewed the manuscript. N.A.J., M.B., B.W. and B.M. prepared the samples, performed sample sorting and $\mathrm{COO}$ analysis and contributed to the text. A.B.-W. and J.J.S. collected and prepared constitutional DNA samples. K.L.M., R.C. S.L. M.F. and S.J. generated de novo assemblies and identified mutations. M.K., S.R., M.G., O.Y. and E.Y.Z. wrote software and contributed to figures. R.D.C. performed copy number analysis and produced a figure and S.B.-N. performed confirmatory FISH experiments. Y.Z. and A.T. produced the sequencing libraries. I.B., R.H., S.J.M.J., R.M., J.S. and M.H. contributed to the development of experimental and analytical protocols. L.R. provided materials and reviewed the manuscript

Author Information The SRA accession number for the submission of the data not included in previous publications is SRP001599, which is linked to the dbGAP study accession phs000235.v2.p1. Reprints and permissions information is available at www.nature.com/reprints. This paper is distributed under the terms of the Creative Commons Attribution-Non-Commercial-Share Alike licence, and is freely available to all readers at www.nature.com/nature. The authors declare no competing financial interests. Readers are welcome to comment on the online version of this article at www.nature.com/nature. Correspondence and requests for materials should be addressed to M.A.M. (mmarra@bcgsc.ca). 